A 76-year-old man was referred to our endoscopic unit with hematochezia and anemia. He had been receiving palliative treatment, including radiation therapy, for a primary stage IV parotid gland cancer. Colonoscopy showed that the mucosa of the terminal ileum had prolapsed into the colonic lumen, and a flat elevated lesion, $8 \mathrm{~mm}$ in diameter and mimicking a small flat adenoma, was seen on the prolapsed ileal mucosa (Figure 1). On palpation with biopsy forceps, the lesion felt more solid and harder than an ordinary adenoma. An endoscopic biopsy sample was sent for histological evaluation, and the findings were consistent with a metastatic parotid gland cancer (Figure 2 ), the histological features being quite similar to those of the primary tumor biopsied previously. No definite source of bleeding was detected on upper and lower gastrointestinal endoscopic examinations. Palliative treatment was continued and the patient died 4 months later.

This case of ileal metastasis from a parotid gland cancer appears to be the first of its kind to have been reported. As the presence of distant metastasis from head and neck cancer is associated with a poor prognosis, treatment is usually performed in a palliative setting [1]. A secondary gastrointestinal tumor detected in the early phase of the disease has never been reported before. Generally among gastrointestinal metastases, the small intestine is the most common site of secondary involvement of various cancers; metastases elsewhere in the gastrointestinal tract are relatively rarer, with the stomach and large intestine involved less frequently than the small intestine, though the esophagus is affected the least often [2]. Modes of gastrointestinal metastasis include hematogenous or lymphogenous spread, direct invasion by a noncontiguous primary carcinoma along the fascia and mesenteric attachments, and dissemination via the peritoneal fluid [2]. Histologically, because the biopsy specimen in this case showed marked lymphatic permeation of tumor cells, the morphology

\title{
An ileal metastasis from a parotid gland cancer that mimicked a flat adenoma
}
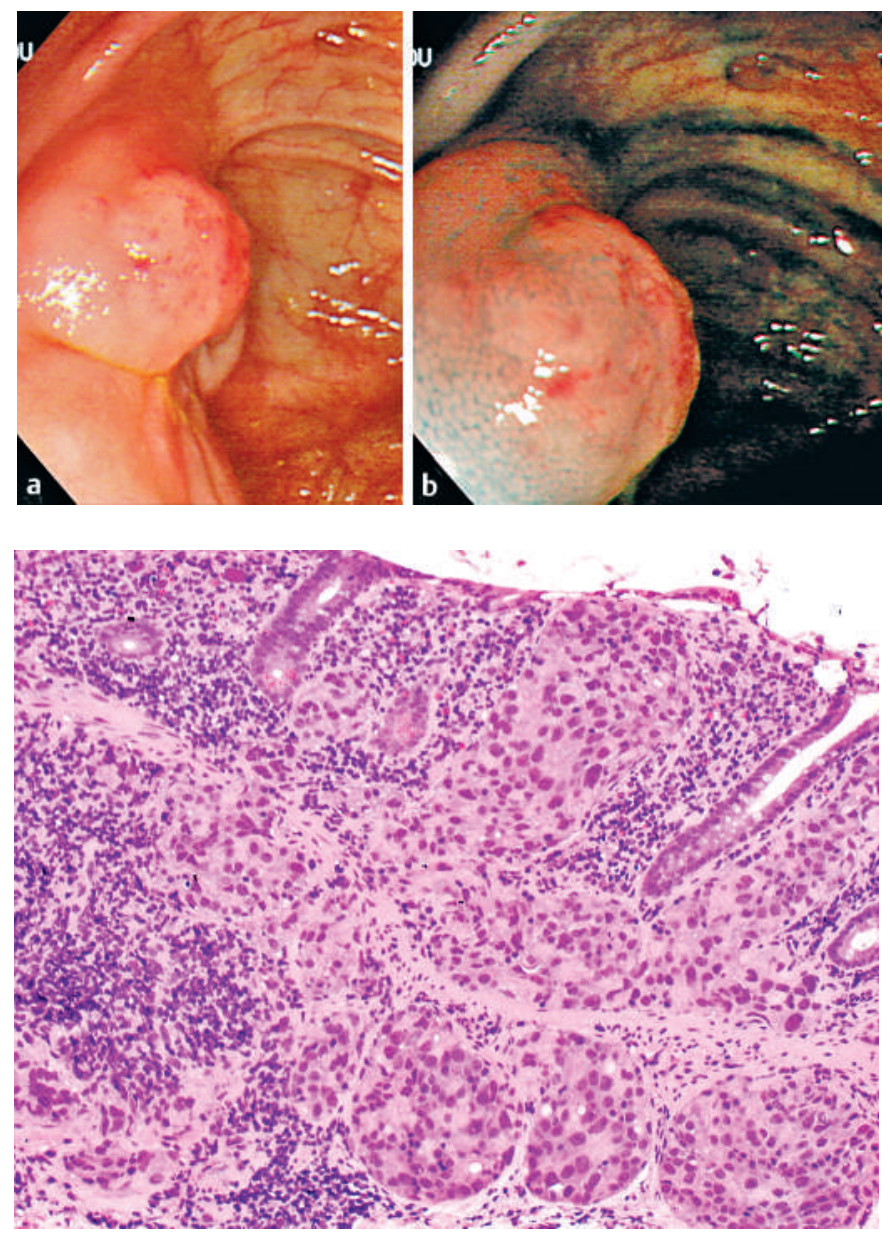

Figure 1 Colonoscopy revealed a small flat elevated lesion on the ileal mucosa that had prolapsed into the colonic lumen, which felt solid and hard on palpation with biopsy forceps (a). Chromoendoscopy using $0.4 \%$ indigo carmine dye clearly defined the margin of the metastatic lesion (b).

Figure 2 Histologically, the biopsy specimen from the flat elevated lesion showed diffuse infiltration of parotid gland carcinoma cells in both the mucosa and the submucosal layer. of the lesion was considered to have been created by lymphogenous spread of solid tumor cells.

\section{Endoscopy_UCTN_Code_CCL_1AC_2AC}

\section{K.I. Fu', , T. Ishikawa², H. Fujii'²,} K. Hirabayashi ${ }^{3}$, S. Igarashi' ${ }^{3}$, Y. Kaji ${ }^{1}$

${ }^{1}$ Department of Radiology, Dokkyo University School of Medicine, Mibu, Shimotuga, Tochigi, Japan

2 Department of Diagnostic Imaging, Division of Endoscopy, Tochigi Cancer Center Hospital, Utsunomiya, Tochigi, Japan

3 Department of Pathology, Tochigi Cancer Center Hospital, Utsunomiya, Tochigi, Japan.

\section{References}

${ }^{1}$ Schwentner I, Obrist P, Thumfart W, Sprinzl G. Distant metastasis of parotid gland tumors. Acta Otolaryngol 2006; 126: 340-345 2 Caramella E, Bruneton JN, Roux P et al. Metastases of the digestive tract: report of 77 cases and review of the literature. Eur J Radiol 1983; 3: $331-338$

\section{Corresponding author}

\section{K.I. Fu, M. D., Ph. D.}

Department of Radiology

Dokkyo University School of Medicine

880 Kitakobaysashi · Mibu · Shimotuga

Tochigi 321-0293 · Japan

Fax: $\quad+81-282-86-5678$

Email: fukuangi@hotmail.com 